\author{
Lt Col KM RAl ${ }^{*}$, Col KK MAUDAR ${ }^{+}$
}

MJAFI 1997; 53 : 66-67

$\mathbf{P}$ rostanoids are prostaglandin analogues which are potent vasodilators and inhibitors of platelet aggregation. This property has been utilized in treatment of limb ischemia where direct arterial surgery or angioplasty is not possible. Prostaglandin El (PGE1) was first tried in occlusive arterial disease in 1973 and prostacyclin a few years later. Iloprost, a stable prostacyclin analogue was introduced subsequently. Prostanoids have a short half-life and therefore intravenous or intra-arterial route is required for their administration. This limitation led to development of two orally active compounds, cicaprost and beraprost, and their initial reports are encouraging [1].

\section{Mechanism of action}

Prostanoids cause arteriolar and veno-dilatation causing increase in skin temperature and facial flushing. They are also potent inhibitors of platelet aggregation and activation, the effect lasting for 100 minutes or more after the end of infusion. While these two are the most well documented effects, they have other beneficial effects too; they inhibit activation of leukocytes, erythrocyte aggregation is inhibited and deformability increased, mitotic activity and proliferation of smooth muscles of vessel wall is inhibited and they also have a 'cytoprotective effect' whereby the injured cell membrane is prevented from being irreversibly damaged. These actions lead to an increase in supply of oxygen and nutrients as well as their better utilization within the ischemic tissue [2].

\section{Clinical Pharmacology}

The majority of prostanoids require intravenous or intra-arterial infusion as they have a very short half-life. Prostacyclin infusion in the dose of 2-5 $\mathrm{ng} / \mathrm{kg} / \mathrm{min}$ causes arteriolar vasodilatation, tachy- cardia, increase in skin temperature and facial flushing. These cardiovascular effects are shortlived as compared to the effects on platelets which last for almost 2 hours after the end of the infusion. Headache, colicky abdominal pain, ill-defined sense of unease, restlessness, weakness and nausea and vomiting are the reported side-effects but are infrequent. Higher infusion rates of intravenous prostanoids may cause sudden hypotension. Prostanoids are contraindicted where the antiplatelet effect may increase the risk of haemorrhage, e.g. active peptic ulcer, trauma, intracranial haemorrhage. Severe coronary heart disease, congestive heart failure, and arrhythmias are other contraindications, as is pregnancy and lactation.

\section{Clinical use}

Iloprost (llomedin) in the dose of $2-4$ $\mathrm{ng} / \mathrm{kg} /$ minute intravenously for 6-8 hours every day for 4 weeks has been extensively used in treatment of critical limb ischemia (rest pain in the limb or non-healing ulcers) where direct arterial surgery is not feasible. This presentation is common in late stages of atherosclerotic occlusive arterial disease as well as in thromboangiitis obliterans (Buerger's disease), an occlusive arterial disease involving the small and medium size arteries which is common in India, Japan and Israel. The comparable dose of PGEl is $40-60 \mu \mathrm{g}$ per day for 3-4 weeks. A recent meta-analysis of six randomized controlled trials [2] showed that patients treated with iloprost have a significant beneficial effect $(p<0.05)$ in terms of pain relief and healing of ulcers as compared to placebo, naftidofuryl or pentoxyfylline. The probability of being alive with both legs (i.e. avoiding an amputation) at six months was also considerably more $(p<0.05)$ with 
iloprost. In general, for critical ischemia the response rates vary between 40-65 per cent. At present it is not possible to predict which of these patients will respond to prostanoid therapy. Prostanoids are also very useful in the treatment of severe secondary Raynouds disease unresponsive to other conventional pharmacotherapy [3]. Initial reports in the management of acute limb ischemia following intra-arterial drug injection are also encouraging [4]. They have also been tried as an adjunct to infragenicular bypass surgery [1] and following microvascular skin-flap reconstruction [5] but their efficacy for these conditions has not been proven by randomized controlled trials.

\section{REFERENCES}

I. Norgren $L$. The place of prostanoids in the treatment of critical limb ischemia. In: Norgren L, Bocclaon H, editors
Critical Ischemia. Susex Cambridge Medical Publications, 1992; Vol 3, Supplement 1: 38-43.

2. Loosemore TM, Chalmers TC, Dormandy JA. A metaanalysis of randomized placebo control trials in Fontaine stages III and IV peripheral occlusive arterial disease. Int Angiol 1994; 13: 33-42.

3. Rademaker M, Cooke ED, Almond NE, et al. Comparison of intravenous infusions of iloprost and oral nifedipine in treatment of Raynaud's phenomenon in patients with systemic sclerosis: a double-blind randomized study. BMJ 1989; 29: 561-4.

4. Andreev A, Kavrokov T, Petkov D, Penkov P. Severe acute hand ischemia following accidental intranterial drug injection, successfully treated with thrombolysis and intraarterial iloprost infusion. Angiology 1995; 46: 963-7.

5. Forman DL, Shah DK, Zhwang WX, et al. Evaluation of a continuous systemic infusion of iloprost, a stable PGI2 analogue on the survival of skin flaps. J Reconstr Microsurg 1995; 11: 339-44. 